\title{
AN OPTIMAL CONTROL FORMULATION FOR INVISCID INCOMPRESSIBLE IDEAL FLUID FLOW
}

Proc. $C D C, \mathbf{3 9}, \mathbf{1 2 7 3 - 1 2 7 9}$

\author{
Anthony M. Bloch ${ }^{1}$ \\ Department of Mathematics \\ University of Michigan \\ Ann Arbor MI 48109 \\ abloch@math.lsa.umich.edu \\ Darryl D. Holm ${ }^{3}$ \\ Mathematical Modeling and Analysis \\ Theoretical Division \\ Los Alamos National Laboratory \\ Los Alamos, NM 87545 \\ dholm@lanl.gov
}

\section{Contents}

1 Introduction

2 Inviscid, Incompressible, Fluid Flow

3 Optimal Control Problem

4 Hamiltonian Structure of Extremals

5 Conclusions

6 References impulse density with the velocity. We provide a comparative discussion of the flow equations in their Eulerian and Lagrangian form and describe how these forms occur naturally in the context of optimal control. We demonstrate that the extremal equations corresponding to the optimal control problem for the flow have a natural canonical symplectic structure.

\section{Introduction}

In this paper we consider the Hamiltonian formulation of the equations of incompressible ideal fluid flow from the point of view of optimal control theory. Our goal in this work is to compare the fluid equation arising in this fashion with the symmetric generalized rigid body equations derived in Bloch and Crouch [1996], Bloch, Brockett and Crouch [1997] and Bloch, Crouch, Marsden and Ratiu [1998]. In these papers we showed that a natural control approach led to a form of the rigid body equations on $\mathrm{SO}(n) \times \mathrm{SO}(n)$ rather than on $T^{*} \mathrm{SO}(n)$. In contrast here we show that the optimal control approach leads to a standard formulation of the Euler equations - the so-called impulse equations in their Lagrangian form. A nice survey of the impulse equations in the various forms can be found in Russo and Smereka [1999] (see also Kuz'min [1983], Osledets [1989] and Maddocks and Pego [1995] for related work). Lie-Poisson reduction is an important tool in rationalizing many of these approaches (see e.g. Marsden and Weinstein [1984]). In particular, the Hodge projection to the divergence free vector fields, is a Poisson map since it may be regarded as the dual of the natural inclu-

\footnotetext{
${ }^{1}$ Research partially supported by the NSF and AFOSR.

${ }^{2}$ Work supported in part by NSF and NATO.

${ }^{3}$ Work supported in part by DOE

${ }^{4}$ Research partially supported by NSF and AFOSR.
}

\author{
Peter E. Crouch ${ }^{2}$ \\ Center for Systems Science \\ and Engineering \\ Tempe, AZ 85287 \\ peter.crouch@asu.edu \\ Jerrold E. Marsden ${ }^{4}$ \\ California Institute of Technology \\ Pasadena, CA 91125 \\ marsden@cds.caltech.edu
}


sion (this is a standard result; see Marsden and Ratiu [1994]). Thus, the Hodge projection naturally takes the unconstrained Poisson system to the constrained one. Russo and Smereka and others concern themselves with the numerical aspects of these equations, something we do not consider here.

These impulse equations are not symmetric in the same sense as the symmetric rigid body equations i.e. we do not obtain two symmetric equations evolving on two copies of the diffeomorphism group, but it is possible to get a more symmetric formulation which we intend to discuss in a forthcoming publication and that we mention briefly in the conclusions.

In the remainder of the introduction we recall the standard and symmetric rigid body equations.

We recall from Manakov [1976] and Ratiu [1980] that the left invariant generalized rigid body equations on $\mathrm{SO}(n)$ may be written as

$$
\begin{aligned}
\dot{Q} & =Q \Omega \\
\dot{M} & =[M, \Omega],
\end{aligned}
$$

where $Q \in \mathrm{SO}(n)$ denotes the configuration space variable (the attitude of the body), $\Omega=Q^{-1} \dot{Q} \in \mathfrak{s o}(n)$ is the body angular velocity, and

$$
M:=J(\Omega)=\Lambda \Omega+\Omega \Lambda \in \mathfrak{s o}(n)
$$

is the body angular momentum. Here $J: \mathfrak{s o}(n) \rightarrow$ $\mathfrak{s o}(n)$ is the symmetric (with respect to the above inner product) positive definite operator defined by

$$
J(\Omega)=\Lambda \Omega+\Omega \Lambda
$$

where $\Lambda$ is a diagonal matrix satisfying $\Lambda_{i}+\Lambda_{j}>0$ for all $i \neq j$. For $n=3$ the elements of $\Lambda_{i}$ are related to the standard diagonal moment of inertia tensor $I$ by $I_{1}=\Lambda_{2}+\Lambda_{3}, I_{2}=\Lambda_{3}+\Lambda_{1}, I_{3}=\Lambda_{1}+\Lambda_{2}$.

The equations $\dot{M}=[M, \Omega]$ are readily checked to be the Euler-Poincaré equations on $\mathfrak{s o}(n)$ for the Lagrangian

$$
l(\Omega)=\frac{1}{2}\langle\Omega, J(\Omega)\rangle .
$$

The left invariant symmetric rigid body system is given by the first order equations

$$
\begin{aligned}
& \dot{Q}=Q \Omega \\
& \dot{P}=P \Omega
\end{aligned}
$$

where $\Omega$ is regarded as a function of $Q$ and $P$ via the equations

$$
\Omega:=J^{-1}(M) \in \mathfrak{s o}(n) \text { and } \quad M:=Q^{T} P-P^{T} Q .
$$

These equations can be derived from the following optimal control problem:
Definition 1 Let $T>0, Q_{0}, Q_{T} \in \mathrm{SO}(n)$ be given and fixed. Let the rigid body optimal control problem be given by

$$
\min _{U \in \mathfrak{s o}(n)} \frac{1}{4} \int_{0}^{T}\langle U, J(U)\rangle d t
$$

subject to the constraint on $U$ that there be a curve $Q(t) \in \mathrm{SO}(n)$ such that

$$
\dot{Q}=Q U \quad Q(0)=Q_{0}, \quad Q(T)=Q_{T}
$$

Proposition 2 The rigid body optimal control problem 1 has extremal evolution equations (1.1) where $P$ is the costate vector given by the maximum principle.

The optimal controls in this case are given by

$$
U=J^{-1}\left(Q^{T} P-P^{T} Q\right) .
$$

In $\S 3$ we derive the impulse equations for fluid flow from the optimal control point of view.

\section{Inviscid, Incompressible, Fluid Flow}

In this section we introduce the usual dynamics for inviscid, incompressible fluid flow, impulse density and the vorticity dynamics. The basic equations we consider are:

$$
\begin{aligned}
\frac{\partial v}{\partial t}+(v \cdot \operatorname{grad}) v & =-\operatorname{grad} p ; \quad \operatorname{div} v=0 \\
x \in \Omega ; \quad v & =v(x, t), \quad p=p(x, t) .
\end{aligned}
$$

We assume, for simplicty only that the flow is in all of space or in a periodic box so we do not need to deal with boundary conditions. This is not an essential restriction.

Here, $v$ is the fluid velocity and $p$ is the pressure. We introduce the impulse density $z$,

$$
z=v+\operatorname{grad} k .
$$

where $k$ is an arbitrary scalar field, $k=k(x, t)$. Notice that the preceding equation gives the (Helmholtz)Hodge decomposition of $z$. In other words, the projection of $z$ to $v$ is the Hodge projection of $z$. We return to this important remark in the conclusions to gain deeper insight into what is going on with the calculations to follow.

Take the time derivative of (2.2) to get

$$
\frac{\partial z}{\partial t}-v \times \operatorname{curl} z=\operatorname{grad} \Lambda, \quad \operatorname{div} v=0
$$

where

$$
\Lambda=\frac{\partial k}{\partial t}-p-\frac{1}{2} v \cdot v
$$

$\Lambda$ is called the gauge. Any choice of gauge is possible, but to be concrete, we consider the "geometric gauge" $\Lambda=-v \cdot z$. 
With this choice

$$
\frac{\partial z}{\partial t}+(v \cdot \operatorname{grad}) z+(\operatorname{grad} v)^{T} z=0, \quad \operatorname{div} v=0
$$

and $k$ is now fixed by the equation

$$
\frac{d k}{d t}=p-\frac{1}{2} v \cdot v
$$

Let $\mathfrak{z}=\sum_{i} z_{i} d x_{i}(=z \cdot d x)$ be the one form corresponding to $z$. Then one can easily show that

$$
\mathcal{L}_{\mathrm{v} \mathfrak{z}}=\left(z_{*} v+v_{*}^{T} z\right) \cdot d x .
$$

Thus the impulse density is governed by:

$$
\frac{\partial \mathfrak{z}}{\partial t}+\mathcal{L}_{\mathrm{v} \mathfrak{z}}=0, \quad \operatorname{div} v=0 .
$$

Hence by applying the exterior differential operator we obtain

$$
\frac{\partial}{\partial t} d \mathfrak{z}+\mathcal{L}_{\mathrm{v}} d_{\mathfrak{z}}=0, \quad \operatorname{div} v=0 .
$$

Lemma $3 w=\operatorname{curl} z=\operatorname{curl} v$ satisfies the vorticity equation:

$$
\frac{\partial w}{\partial t}+[v, w]=0
$$

Proof $\quad d \mathfrak{z}=\sum_{i} d z_{i} \wedge d x_{i}=\sum_{i j}(\hat{\operatorname{curl} z} z)_{i j} d x_{i} \otimes d x_{j}$, where $\hat{a} b=a \times b$. We may now compute:

$$
\mathcal{L}_{\mathrm{v}} d \mathfrak{z}=\sum_{i j}[v, \operatorname{curl} z]_{i j}^{\wedge} d x_{i} \otimes d x_{j}
$$

We now quickly review the two coordinate systems associated with the fluid system. We denote the Lagrange or material variables by $X_{i}$ and the Euler or spatial variables by $x_{i}$, and set

$$
x_{i}=\phi_{i}(X, t), \quad 1 \leq i \leq 3 .
$$

We assume $\phi: \Omega \rightarrow \Omega$ is a volume preserving diffeomorphism, with Jacobian equal to unity, $\left|\phi_{*}\right|=1$.

Let $v(x, t)=$ spatial velocity, so that

$$
\frac{\partial x_{i}}{\partial t}=v_{i}(x, t)
$$

Thus $V(X, t)=v(\phi(X, t))$ is the material velocity. Hence

$$
\frac{\partial \phi}{\partial t}(X, t)=v(\phi(X, t))
$$

or

$$
\frac{\partial \phi}{\partial t}=v \circ \phi
$$

We note the "right invariance" of this system and its evolution on the "Group" $G=\operatorname{Diff}_{\mathrm{vol}}(\Omega)$ of volume preserving diffeomorphisms of $\Omega$. Setting

$$
\langle a, b\rangle=\int_{\mathbb{R}^{3}} a^{T}(x, t) b(x, t) d x,
$$

and using $\left|\phi_{*}\right|=1$, we obtain the identity

$$
\langle a \circ \phi, b\rangle=\left\langle a, b \circ \phi^{-1}\right\rangle .
$$

With this introduction we may introduce the total vorticity equations:

$$
\frac{\partial \phi}{\partial t}=v \circ \phi ; \quad \frac{\partial w}{\partial t}=[w, v]: \operatorname{div} v=0 .
$$

It is interesting to compare these equations with the right invariant Euler equations for the rigid body:

$$
\begin{gathered}
\dot{Q}=\Omega Q ; \quad \dot{M}=[\Omega, M] \\
{[\Omega, M]=\Omega M-M \Omega\left(\begin{array}{c}
=[M, \Omega] \text { interpreted } \\
\text { as vector fields }
\end{array}\right) .}
\end{gathered}
$$

These Euler equations are Hamiltonian on $T^{*} \mathrm{SO}(3)$, with the canonical symplectic structure. The equivalent statements about (2.7) have been well studied, (see references in the introduction). However, the derivation of the symmetric version as in (1.1) provides our motivation for this new study.

\section{Optimal Control Problem}

In this section we introduce an optimal control problem and discuss the corresponding extremals. The problem can be posed as:

$$
\min _{v(\cdot)} \frac{1}{2} \int_{0}^{T}\langle v, v\rangle d t
$$

subject to:

$$
\operatorname{div} v=0 ; \quad \frac{\partial \phi}{\partial t}=v \circ \phi
$$

and

$$
\phi(X, 0)=\phi_{0}(X), \quad \phi(X, T)=\phi_{T}(X) \text { fixed },
$$

and, for flow in all of space, suitable conditions at infinity.

This optimal control problem is of course identical to the standard Hamilton principle for ideal fluid mechanics. However our goal here is to analyze it from the point of view of the Pontryagin maximum principle.

We solve this problem by introducing Lagrange multipliers and the cost

$J(v, \phi, \pi, k)=\int_{0}^{T}\left(\left\langle\pi, v \circ \phi-\frac{\partial \phi}{\partial t}\right\rangle-\frac{1}{2}\langle v, v\rangle+\langle k, \operatorname{div} v\rangle\right) d t$

The problem (3.1) may be recast as: $\min J$, subject to $\operatorname{div} v=0, \frac{\partial \phi}{\partial t}=v \circ \phi$, and boundary conditions.

We may prove the following result: 
Theorem 4 The extremals of problem (3.1) are given by

$$
\begin{aligned}
\frac{\partial \pi}{\partial t} & =-\left(v_{*} \circ \phi\right)^{T} \pi, \quad \frac{\partial \phi}{\partial t}=v \circ \phi \\
v & =\pi \circ \phi^{-1}-\operatorname{grad} k, \quad \operatorname{div} v=0 .
\end{aligned}
$$

\section{Sketch Proof}

$$
\begin{aligned}
\delta J= & \int_{0}^{T}\left(\left\langle\pi, \delta v \circ \phi+\left(v_{*} \circ \phi\right) \delta \phi-\frac{\partial}{\partial t} \delta \phi\right\rangle-\langle v, \delta v\rangle\right. \\
& +\langle k, \operatorname{div} \delta v\rangle) d t \\
= & \int_{0}^{T}(\langle\pi, \delta v \circ \phi\rangle-\langle v, \delta v\rangle+\langle k, \operatorname{div} \delta v\rangle) d t \\
& +\int_{0}^{T}\left\langle\pi,\left(v_{*} \circ \phi\right) \delta \phi-\frac{\partial}{\partial t} \delta \phi\right\rangle d t
\end{aligned}
$$

Noting that $\delta v(\infty, t)=\delta \phi(x, 0)=\delta \phi(x, T)=0$, we obtain

$$
\begin{aligned}
\delta J= & \int_{0}^{T}\left\langle\pi \circ \phi^{-1}-v-\operatorname{grad} k, \delta v\right\rangle d t \\
& +\int_{0}^{T}\left\langle\left(v_{*} \circ \phi\right)^{T} \pi+\frac{\partial \pi}{\partial t}, \delta \phi\right\rangle d t .
\end{aligned}
$$

The system (3.2) follows immediately.

We note that the system (3.2) should be interpreted in terms of Lagrange and Euler variables in the form

$$
\begin{aligned}
& \frac{\partial \pi}{\partial t}(X, t)=-\left(\frac{\partial v}{\partial x}(\phi(X, t), t)\right)^{T} \pi(X, t), \\
& \frac{\partial \phi}{\partial t}(X, t)=v(\phi(X, t))
\end{aligned}
$$

and

$$
v(x, t)=\pi \circ \phi^{-1}(x, t)-\operatorname{grad} k(x, t) .
$$

We now study the Hamiltonian for the extremal flow. Employing the maximum principle we know that the Hamiltonian corresponding to the problem (3.1) is

$$
H(\pi, \phi)=\langle\pi, v \circ \phi\rangle-\frac{1}{2}\langle v, v\rangle .
$$

We introduce the vector potential for $v$

$$
v=\operatorname{curl} \psi ; \quad \operatorname{div} \psi=0,
$$

(and $\psi \rightarrow 0$ at infinity in all of space). Thus

$$
\omega=\operatorname{curl} v=\operatorname{curl} \operatorname{curl} \psi=-\Delta \psi+\operatorname{grad} \operatorname{div} \psi=-\Delta \psi,
$$

so

$$
\omega=-\Delta \psi ; \quad \Delta=\text { Laplacian; }
$$

Thus $\psi=A \omega$ where $A$ is an integral operator. From these identities we may write:

$$
\begin{aligned}
H(\pi, \phi) & =\left\langle\pi \circ \phi^{-1}, \operatorname{curl} \psi\right\rangle-\frac{1}{2}\langle v, \operatorname{curl} \psi\rangle \\
& =\left\langle\operatorname{curl} \pi \circ \phi^{-1}, \psi\right\rangle-\frac{1}{2}\langle\operatorname{curl} v, \psi\rangle
\end{aligned}
$$

But

$$
v=\pi \circ \phi^{-1}-\operatorname{grad} k
$$

so

$$
\operatorname{curl} v=\operatorname{curl} \pi \circ \phi^{-1}
$$

and

$$
\begin{aligned}
H(\pi, \phi) & =\frac{1}{2}\left\langle\operatorname{curl} \pi \circ \phi^{-1}, \psi\right\rangle \\
& =\frac{1}{2}\left\langle\pi \circ \phi^{-1}, v\right\rangle \\
& =\frac{1}{2}\langle\omega, A \omega\rangle \\
& =\frac{1}{2}\left\langle\operatorname{curl} \pi \circ \phi^{-1}, A \operatorname{curl} \pi \circ \phi^{-1}\right\rangle .
\end{aligned}
$$

We now compute along extremals (3.2)

$$
\frac{\partial}{\partial t} \sum_{i} \pi_{i}(X, t) d \phi_{i}(X, t)=0
$$

or

$$
\frac{\partial}{\partial t} \sum_{i} \pi_{i} d \phi_{i}=0
$$

Hence

$$
\frac{\partial}{\partial t} \sum_{i} d \pi_{i} \wedge d \phi_{i}=0
$$

Thus the "canonical two form"

$$
\sum_{i} d \pi_{i} \wedge d \phi_{i}=\sum_{i j k} \frac{\partial \pi_{i}}{\partial X_{j}} \frac{\partial \phi_{i}}{\partial X_{k}} d X_{j} \wedge d X_{k}
$$

is constant along extremals. We now have the following critical result.

Lemma 5 Let $z=\pi \circ \phi^{-1}$. Along extremals (3.2)

$$
\frac{\partial z}{\partial t}+z_{*} v+v_{*}^{T} z=0
$$

Thus $\mathfrak{z}=z \cdot d x$ satisfies

$$
\frac{\partial \mathfrak{z}}{\partial t}+\mathcal{L}_{\mathrm{v} \mathfrak{z}}=0, \quad \frac{\partial \phi}{\partial t}=v \circ \phi
$$

It follows that we have recovered the evolution of the impulse density of the fluid flow, equation (2.4). Note that $H=\frac{1}{2}\langle z, v\rangle$, where $-z \cdot v=\Lambda$ is the geometric gauge. The following result relates $z$ to the canonical two form. 
Lemma $6 \phi^{-1 *} \sum_{i} d \pi_{i} \wedge d \phi_{i}=\sum_{i} d z_{i} \wedge d x_{i}=d \mathfrak{z}$.

Substituting the relation

$$
z=v+\operatorname{grad} k
$$

into the system (3.7) recovers the system (2.1) where

$$
\frac{d k}{d t}=p-\frac{1}{2} \text { v.v. }
$$

However $k$ is not arbitrary, since it is determined by the extremal system (3.2). In fact

$$
\operatorname{div} z=\operatorname{div} \pi \circ \phi^{-1}=\operatorname{div} \operatorname{grad} k=\Delta k .
$$

Thus,

$$
\begin{aligned}
& k=A \operatorname{div} z=A \operatorname{div} \pi \circ \phi^{-1} . \\
& v=\pi \circ \phi^{-1}-\operatorname{grad} A \operatorname{div} \pi \circ \phi^{-1} .
\end{aligned}
$$

Hence the pressure $p$ is also determined by the flow.

Lemma 7 By augmenting the cost functional in the optimal control problem (3.1) by a potential function $\eta$

$$
\int_{0}^{T}\left(\frac{1}{2}\langle v \cdot v\rangle-\int_{\Omega} \eta \circ \phi\right) d t
$$

the extremal flow satisfies the system (2.1) with the pressure determined by

$$
\frac{d k}{d t}=p-\eta-\frac{1}{2} v \cdot v .
$$

$k$ and $v$ are determined from (3.9).

Thus any pressure $p$ may be obtained via a suitable potential $\eta$.

\section{Hamiltonian Structure of Extremals}

We now briefly explore the Hamiltonian nature of (2.1) and the extremal equations (3.2). If $u$ is a smooth function of $x$ and $t$, and $h[u]$ is a function of $x, t$ and the jet of $u$, let

$$
H[u]=\int_{\mathbb{R}^{3}} h[u] d x .
$$

Define

$$
\delta H[u]=\int_{\mathbb{R}^{3}}\left\langle\frac{\delta H(u)}{\delta u}, \delta u\right\rangle d x .
$$

We have the following result:

Theorem 8 For the Hamiltonian (3.6)

$$
\frac{\delta H}{\delta \pi}(\pi, \phi)=v \circ \phi ; \quad \frac{\partial H}{\partial \phi}(\pi, \phi)=\left(v_{*} \circ \phi\right)^{T} \pi .
$$

Thus the extremal equations (3.2) may be written as

$$
\frac{\partial \pi}{\partial t}=-\frac{\delta H}{\delta \phi} ; \quad \frac{\partial \phi}{\partial t}=\frac{\delta H}{\delta \pi} .
$$

These equations are canonical with respect to the natural symplectic form on $L_{2}\left(\mathbb{R}^{3}: \mathbb{R}^{3}\right) \times L_{2}\left(\mathbb{R}^{3}: \mathbb{R}^{3}\right)$

$$
\omega\left(\left(X_{1}, Y_{1}\right),\left(X_{2}, Y_{2}\right)\right)=\int_{\mathbb{R}^{3}}\left(Y_{2} \cdot X_{1}-X_{2} \cdot Y_{1}\right) d x
$$

Thus we have expressed the extremal equations (3.2) in terms of a canonical Hamiltonian system.

\section{Conclusions}

As described earlier, the Euler (impulse) equations (3.2) are not quite in the symmetric form that we obtain in the rigid body setting - i.e. we do not get a symmetric flow on two copies of the diffeomorphism group. However, it is possible to extend the analysis to this setting by factoring $\pi$ as

$$
\pi=r \circ \psi,
$$

where

$$
\frac{\partial \psi}{\partial t}=v \circ \psi
$$

and $\psi$ evolves on the diffeomorphim group. Thus we do get symmetric equations for $\phi$ and $\psi$ coupled to an interesting radial equation for $r$. This also has an analogue in the finite-dimensional setting - one allows $P$ to be in $G l(n)$ and considers the polar decomposition $P=R K$ where $R$ is symmetric positive definite and $K$ lies in $\mathrm{SO}(n)$. We shall describe the details of this analysis in a forthcoming publication.

In addressing these issues, a deeper understanding of both the Hamiltonian and variational structure as well as the geometry is needed. For example, we can obtain more insight into some of the calculations done in this paper as follows. Consider the Hodge projection $\mathbb{P}: \mathfrak{X} \rightarrow \mathfrak{X}_{\mathrm{vol}}$ taking a vector field $z$ to its divergence free part parallel to the boundary. As we have mentioned in the introduction, using the $L_{2}$ pairing, this map is a Poisson map. Taking the $L_{2}$ kinetic energy as the Hamiltonian on the unconstrained space $\mathfrak{X}$ as well as on the constrained space $\mathfrak{X}_{\mathrm{vol}}$, we conclude that the corresponding Hamiltonian systems with their Lie Poisson bracket structures are mapped one to the other (including integral curves) by the Hodge projection. This simple remark is, in fact, the essense of what is going on in relaxing the divergence free constraints and in relating the Hamiltonian structure in the formalism of Osledets, Buttke, and Kusmin. We have, in fact, shown some aspects of this remark in the above direct calculations. A deeper problem, to which we will return in other work, is to carry this out in material representation, where one needs a nonlinear Hodge decomposition, 
similar to the Moser decomposition (a diffeomorphism group analogue of the polar decomposition) discussed in Ebin and Marsden [1970]. Many of these issues are addressed in work of Brenier; see, eg, Brenier [1999].

Acknowledgement: We would like to thank Peter Smereka for useful conversations.

\section{References}

Benjamin T. Brooke [1984] Impulse, Flow Force and Variational Principles, IMA Journal of Applied Mathematics 32, 3-68.

Bloch, A.M., R. W. Brockett, and P.E. Crouch [1997] Double bracket equations and geodesic flows on symmetric spaces. Comm. Math Phys 187,357373.

Bloch, A. M. and P. E. Crouch [1996] Optimal control and geodesic flows. Systems and Control Letters 28, 65-72.

Bloch, A. M., P. E. Crouch J.E. Marsden and T.S. Ratiu [1998] Discrete rigid body dynamics and optimal control Proceedings of the 37th CDC, IEEE, 2249-2254.

Bloch, A.M., P.S. Krishnaprasad, J.E. Marsden and T.S. Ratiu [1994] Dissipation induced instabilities, Ann. Inst. H. Poincaré, Analyse Nonlineare 11, 37-90.

Brenier, Y. [1999] Minimal geodesics on groups of volume-preserving maps and generalized solutions of the Euler equations. Comm. Pure Appl. Math. 52, 411-452.

Buttke, T.F. [1993] Velocity methods: Lagrangian numerical methods which preserve the Hamiltonian structure of incompressible fluid flow. In Vortex Flows and Related Numerical Methods (ed. J.T. Beale, G.H. Cottet and S. Hueberson), Kluwer.

Buttke, T.F. and A.J. Chorin [1993], Turbulence calculations in magentization variables, Appl. $\mathrm{Nu}$ mer. Maths. 12, 47.

Ebin, D.G. and J.E. Marsden [1970] Groups of diffeomorphisms and the motion of an incompressible fluid, Ann. Math. 92, 102-163.

Hamel, G. [1904] Die Lagrange-Eulerschen Gleichungen der Mechanik, Z. für Mathematik u. Physik 50, 1-57.

Hamel, G. [1949] Theoretische Mechanik, SpringerVerlag.

Holm, D.D. [1983] Magnetic tornadoes: threedimensional affine motions in ideal magnetohydrodynamics, Physica D 8 (1983), 170-182.
Kuz'min, G.A. [1983] Ideal incompressible hydrodynamics in terms of the vortex momentum density, Phys. Let. 96A, 88-90.

Lamb, H. [1932] Hydrodynamics, 6th ed., Cambridge University Press.

Maddocks, J.H. and R.L. Pego [1995] An unconstrained Hamiltonian formulation for incompressible fluid flow Comm. Math. Phys. 170, 207-217.

Manakov, S.V. [1976] Note on the integration of Euler's equations of the dynamics of and $n$ dimensional rigid body. Funct. Anal. and its Appl. 10, 328-329.

Marsden, J.E., and T. Ratiu [1994] Introduction to Mechanics and Symmetry, Texts in Applied Math., 17, Springer-Verlag, 2nd edition, 1999.

Marsden, J.E. and A. Weinstein [1983] Coadjoint orbits, vortices, and Clebsch variables for incompressible fluids, Physica D 7, 305-323.

Osledets, V.I. [1989], On a new way of writing the Navier Stokes equation: The Hamiltonian formalism, Russ. Math. Surveys 44, 210-211.

Ratiu, T. [1980] The motion of the free n-dimensional rigid body. Indiana U. Math. J., 29, 609-627.

Russo, G. and P. Smereka [1999] Impulse formulation of the Euler equation: general properties and numerical methods, J. Fluid Mechanics 391, 189209.

Serrin, J. [1959] in Mathematical Principles of Classical Fluid Mechanics, Vol. VIII/1 of Encyclopedia of Physics, edited by S. Flügge (Springer-Verlag, Berlin), sections 14-15, pp. 125-263.

Smereka, P. [1996] A Vlasov description of the Euler equation, Nonlinearity, 9, 1361. 